\title{
PERSPECTIVES DEMOGRÀFIQUES
}

\section{El insostenible aumento de la inseguridad residencial en España}

\author{
Juan Antonio Módenes, Centre d’Estudis Demogràfics y Departamento de Geografía, Univ. Autòn. Barcelona
}

Tras la crisis económica la inseguridad residencial sigue aumentando en España. Más de la mitad de los hogares encabezados por menores de 45 años teme perder su vivienda a corto plazo. En el contexto europeo los jóvenes españoles temen perder su vivienda por no poder pagarla más a menudo (54\% frente a 33\%) y esta diferencia también se ha incrementado en los últimos años a medida que crecía el alquiler. Esta percepción está justificada. El 42\% de los hogares formados por jóvenes ya viven en alquiler de mercado, la opción más insegura de todas para un colectivo acuciado por la precariedad laboral, dejando atrás la etapa de acceso mayoritario a la propiedad. La probabilidad de que un hogar joven se sienta más inseguro que un europeo es mayor, independientemente del tipo de vivienda en el que habite. Esto tiene que ver con un contexto legal e institucional que perjudica la estabilidad en la vivienda. La inseguridad residencial es una de las principales dimensiones de la inseguridad vital por lo que es urgente tomar medidas para controlarla. Este número de Perspectives Demogràfiques examina los niveles de inseguridad residencial en España y examina sus posibles causas, especialmente la evolución del alquiler. Para ello, analizaremos distintas fuentes que nos acercan al crecimiento reciente del alquiler (Encuesta de Condiciones de Vida) o de la inseguridad residencial percibida (European Quality of Life Survey).

\section{MEDIR LA INSEGURIDAD RESIDENCIAL PERCIBIDA}

Para estudiar el miedo a perder la vivienda, que llamaremos inseguridad residencial subjetiva, iremos más allá de los indicadores habituales de tipo objetivo que miden los estadios más graves de este fenómeno. En efecto, ya disponemos de suficientes datos sobre desahucios y lanzamientos (Méndez, Plaza, 2016) y sobre retrasos en los pagos de las hipotecas o los alquileres. La presión financiera que representa la vivienda se puede aproximar con la relación aritmética entre ingresos y gastos residenciales que aparece en diversas encuestas oficiales. Todos ellos son indicadores extraordinariamente útiles, pero no agotan el análisis de la inseguridad residencial. Hay muchos hogares que nunca sufren retrasos en los pagos, por más que tengan dificultades económicas, ni mucho menos son desahuciados, pero pueden sufrir inseguridad sobre la estabilidad de su estancia en la vivienda. Por ello, es interesante conocer la percepción de los hogares sobre su situación residencial. Se trata de una opinión subjetiva que tiene relación directa con la confianza de las personas en el futuro y sobre la cual se basan otras decisiones, como la de expandir o no la familia.

La encuesta European Quality of Life Survey (EQLS), elaborada por el instituto europeo Eurofound, recoge la opinión de las personas sobre su seguridad en la vivienda. Una de sus preguntas hace referencia a la probabilidad de perder la vivienda en los siguientes 6 meses por no poder pagarla. Las opciones de respuesta en la última oleada de 2016 son 5: de totalmente probable a totalmente improbable. Siguiendo el ejemplo del informe de Eurofound sobre la última edición de la EQLS (Eurofound, 2017), consideramos que todos los hogares que no contestan "Muy improbable" tienen inseguridad residencial subjetiva.

Con esta definición, el 38,3\% de los hogares españoles en 2016 percibían un cierto riesgo de perder su vivienda a corto plazo. Es una cifra elevada que refleja una grave situación estructural en el sistema residencial español. Entre 2011 y 2016, la inseguridad residencia ha crecido del 24,9\% al 38,3\% mientras que en Europa ha crecido del 21,1\% al 24,15. Entre la población joven -hogares encabezados por menores de 45 años- las cifras son más elevadas. La inseguridad residencial ha crecido del 39,7\% al 53,9\% entre 2011 y 2016, muy por encima de la media europea, que lo ha hecho del $29,4 \%$ al $32,7 \%$.

Tras el rápido aumento de la inseguridad residencial en España y la creciente diferencia con Europa destacan dos factores: 1) el incremento del peso de las opciones residenciales más comprometidas financieramente, en especial el alquiler de mercado, en un contexto 
de creciente precariedad sociolaboral (Pareja, Sánchez, 2017); y, 2) un déficit de protección legal, con independencia del tipo de vivienda (Leal, 2015) que se traduce en un exceso de inseguridad. Para ilustrar el primer punto, a continuación se analizan datos sobre la evolución de la vivienda de alquiler en España.

\section{A MÁS ALQUILER, MÁS PRECARIEDAD}

El crecimiento y el estallido posterior de la burbuja inmobiliaria (Bosch, 2018) han transformado las bases fundamentales del sistema residencial español (Módenes, 2014). Uno de los principales cambios que identificamos fue el incremento progresivo del alquiler como vía de acceso a la vivienda entre los jóvenes, aunque los altos porcentajes de propietarios del total de hogares todavía reflejaran la inercia del resto de generaciones.

La Encuesta de Condiciones de Vida (ECV) permite examinar la evolución del alquiler hasta la actualidad. La Figura 1 muestra la evolución de la tenencia residenciad entre los hogares jóvenes, clasificados según la persona de referencia sea menor de 30 o tenga entre 30 y 44 años. La serie se inicia a mediados de los años noventa con datos del Panel de Hogares. En 2017, los hogares cuya persona principal tiene menos de 30 años presentan una distribución de tenencia inédita respecto del pasado reciente. Los propietarios están en minoría (26,5\%). Casi un 50\% vive en alquiler de mercado, mientras que las viviendas cedidas son el 21\%. Esta última categoría refleja la ayuda directa del entorno familiar al acceso a la vivienda. El aumento del alquiler ha sido considerable en los últimos años, también en los hogares adultos-jóvenes (30-44) que han llegado al $25 \%$ de alquiler. La tendencia es muy robusta, cabe esperar que en los próximos años se mantenga y con ello se intensifique la exposición a las vicisitudes del mercado del alquiler de los hogares más jóvenes, dedicados a la consolidación de sus proyectos familiares.

El aumento del alquiler en España y la transformación de la tenencia residencial han ido acompañados en los últimos años de una fuerte precarización del mercado laboral, especialmente visible entre la población joven (Lozano, Rentería, 2018). La Encuesta de Población Activa permite aproximarnos a la precarización laboral de los jóvenes, entendida como la proporción de activos que están en paro o tienen empleos temporales. La precariedad de la oferta de mano de obra alcanzó el máximo en 2013 con el 40\%. A partir de entonces la cifra ha bajado ligeramente y actualmente es del $34 \%$. En el conjunto de la Unión Europea nunca se alcanzó el 21\% y en 2017 está en 19\%. Este indicador superó el 45\% para los jóvenes en edad de formación de hogar en España (25-39 años) y ha descendido algo hasta el 41\% desde el punto álgido de la crisis. Si se busca una definición más estructural de los hogares con problemas sociales, estimando a partir de la EQLS 2016, el 26\% de los hogares de menores de 45 años estaría en riesgo de pobreza o exclusión social (AROPE), por el 22\% del conjunto de la Unión. Por otro lado, Lozano y Rentería (2018) distinguen la evolución creciente estructural de la temporalidad de los contratos que se ha doblado en los últimos 30 años y la evolución cíclica y coyuntural de las tasas de paro. Como resultado las autoras concluyen que la precariedad total se ha ido generalizando y se constituye en un rasgo innato de la evolución vital de los hogares. El aumento del alquiler y la precariedad se unen en un modelo residencial que camina hacia la inseguridad.
FIGURA 1. Evolución reciente de la tenencia residencial de los hogares jóvenes. España (1995-2017)
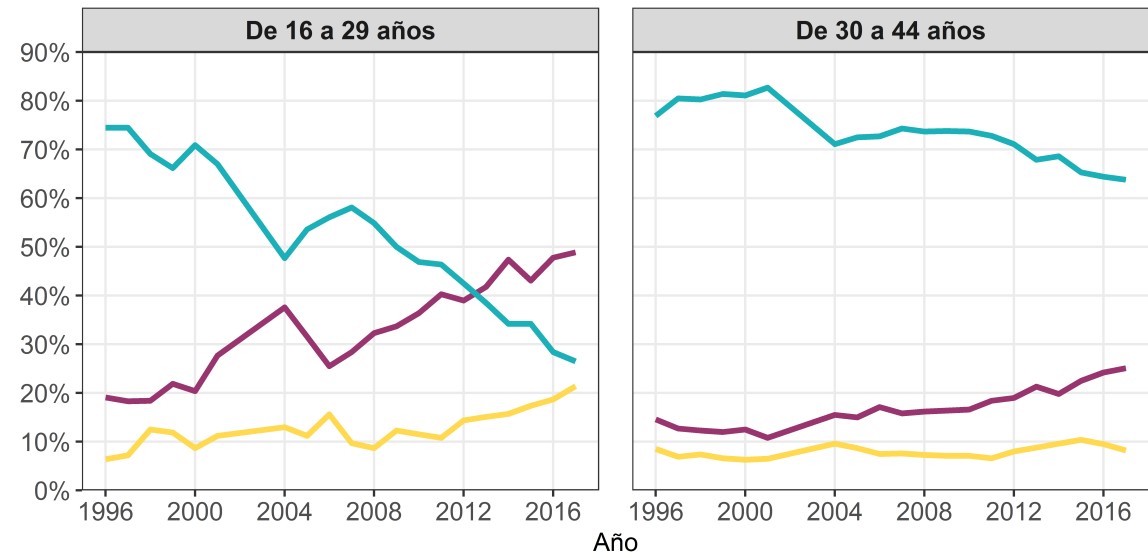

- Alquiler — Propiedad — Viviendas cedidas

Fuente: Elaboración propia a partir del INE, Panel de Hogares (antes de 2004) y Encuesta de Condiciones de Vida (después de 2004)
JOVEN + ALQUILER DE MERCADO = PERCEPCIÓN DE INSEGURIDAD RESIDENCIAL

En la Figura 2 hemos distribuido los hogares jóvenes (<45 años) en función de la tenencia de la vivienda y la percepción de inseguridad residencial inminente, comparando la situación del conjunto de la Unión Europea con la de España. Dos hechos destacan: el sesgo en España hacia las opciones residenciales con mayor compromiso económico (propiedad con hipoteca y alquiler de mercado) y la mayor inseguridad residencial de los españoles, especialmente en las tenencias más frecuentes. Hay que señalar que ya son un porcentaje 
FIGURA 2. Distribución de los hogares jóvenes (<45) por tenencia y percepción de inseguridad residencial. Unión Europea y España
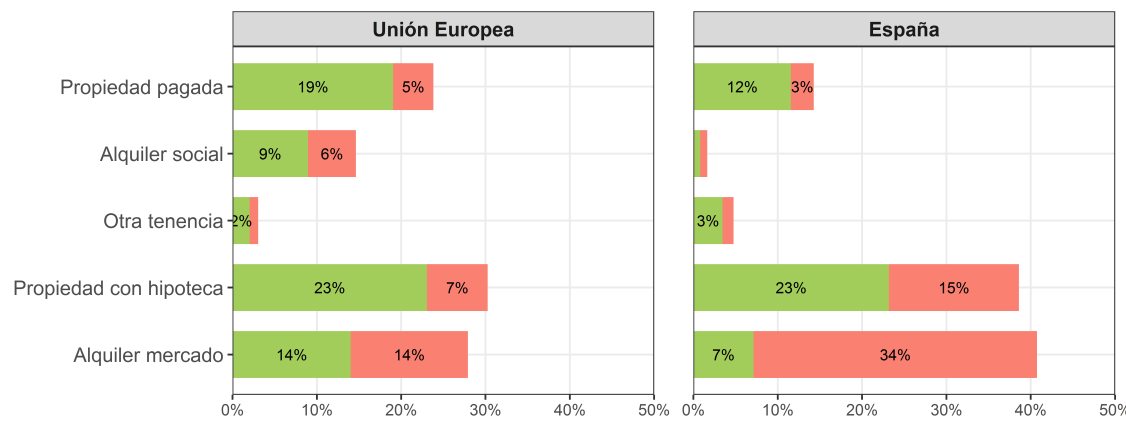

Seguridad residencial Inseguridad residencial

Fuente: Elaboración propia a partir de European Quality of Life Survey 2016

reducido los hogares jóvenes que son propietarios sin pagos pendientes. Algo que era muy habitual en el pasado reciente y constituía uno de los rasgos distintivos del sistema residencial español. Por otro lado, si bien es verdad que en el conjunto de la Unión los hogares en alquiler de mercado sufren un mayor riesgo de inseguridad, en el caso español la inseguridad que perciben los inquilinos jóvenes es casi universal, y mucho mayor que la que perciben los jóvenes hipotecados. Podemos estimar que más de un 80\% de los hogares constituidos por jóvenes que viven en alquiler de mercado perciben inseguridad residencial. Un 70\% de los hogares inseguros jóvenes pagan alquiler. Se puede hablar casi de endogeneidad (o que es lo mismo una cosa que la otra) entre vivir en alquiler y experimentar inseguridad residencial subjetiva.

El caso español sobresale no sólo respecto a la media europea, sino en comparación con los grandes países de la Unión, similares tanto en peso demográfico como en heterogeneidad social, económica y política (Figura 3). Sólo Italia tiene un grado de inseguridad asociada al alquiler de mercado comparable, con la diferencia de que el peso del alquiler en los hogares jóvenes es más elevado en España (41\% frente a 24\%). Francia está en una posición intermedia: más de la mitad de los jóvenes en alquiler de mercado indican inseguridad, aunque solo un tercio del total viven en esta tenencia. Alemania y Reino Unido son los países más seguros para los jóvenes, más aún si viven en alquiler. En el caso de Alemania casi la mitad de los jóvenes habitan en alquiler de mercado que, a la vista de los datos presentados, es una experiencia residencial mucho más segura y estable que la española.

Las diferencias sociales son relevantes e introducen heterogeneidad tanto en la distribución de tenencia como en la percepción de la inseguridad en España (Figura 4). Sin embargo, creemos que ya se puede decir que la inseguridad residencial es transversal. Por un lado, los hogares jóvenes en riesgo de pobreza o exclusión (AROPE) están abocados a vivir en alquiler de mercado (64\%). Su presencia en otras tenencias como la propiedad con o sin hipoteca u otras formas más estables (como la cesión o el alquiler social) es minoritaria, si no residual. Los hogares en alquiler de mercado experimentan inseguridad residencial como algo consustancial de su desarrollo vital (más del 90\%). Las otras formas de tenencia son más seguras pero minoritarias como decíamos. Por otra parte, aunque los hogares de jóvenes sin riesgo social mayoritariamente son propietarios con hipoteca y tienen una percepción de inseguridad más baja, su inseguridad cuando viven en alquiler de mercado, un tercio de ellos, es alta.

\section{PINCHAR LA BURBUJA DE LA INSEGURI- DAD RESIDENCIAL}

En España el alquiler privado ya es la principal vía de acceso a la vivienda de los hogares jóvenes. Dado que acceder a una vivienda en alquiler se ha convertido en la vía de acceso preferente en ámbitos urbanos, podríamos decir que para los jóvenes formar un hogar en los últimos años implica entrar en un estado, no sólo subjetivo, de
FIGURA 3. Percepción de inseguridad residencial de los hogares jóvenes (<45) según viven en alquiler de mercado u otra tenencia. Comparación España con grandes países de la UE. 2016
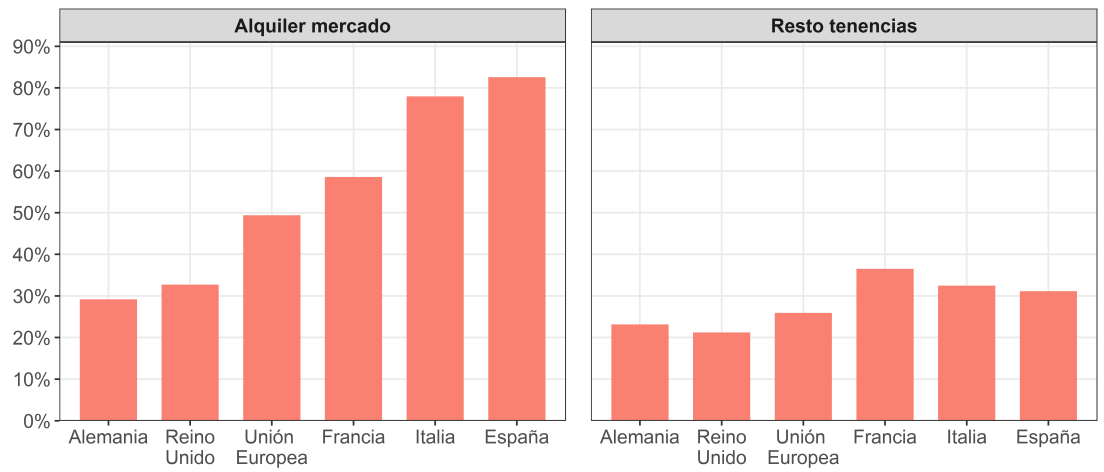

Fuente: Elaboración propia a partir de la European Quality of Life Survey 2016 
FIGURA 4. Distribución hogares jóvenes (<45) por tenencia y percepción de inseguridad residencial según situación de riesgo social (AROPE). España
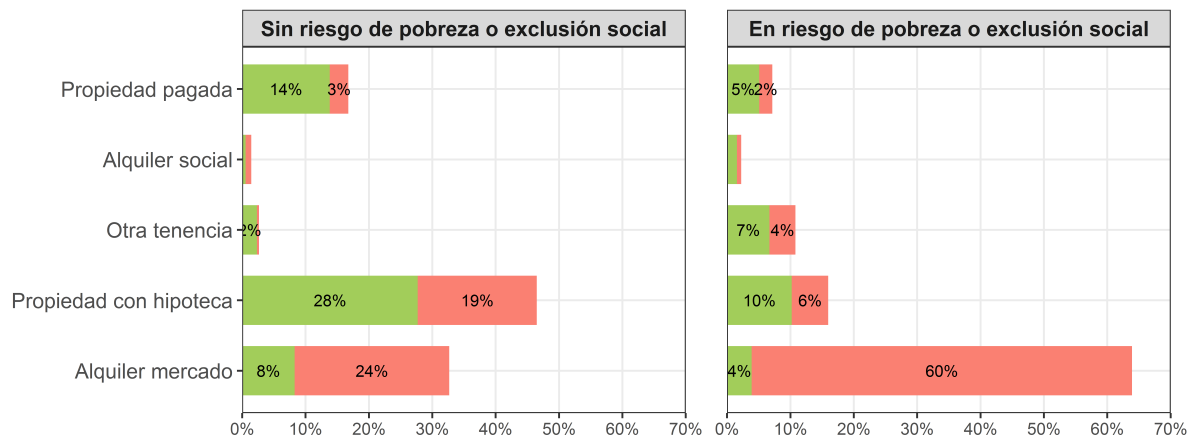

Seguridad residencial

Inseguridad residencial
La inseguridad residencial ha penetrado en capas sociales menos desfavorecidas, las cuales también tienen el alquiler como vía frecuente de acceso a la vivienda. La normalización social de la inseguridad residencial explica el protagonismo mediático en los últimos tiempos de la crisis del alquiler en las grandes ciudades. En este contexto, es urgente tomar medidas que limiten la inseguridad del alquiler de mercado en nuestro sistema residencial. Aceptando que el alquiler está aquí para quedarse, cualquier modificación del contexto institucional que reduzca la inseguridad residencial puede tener un importante inseguridad e inestabilidad residenciales. El auge del alquiler privado ha facilitado el incremento de la inseguridad residencial, dadas las condiciones objetivas de los contratos, su duración y la falta de control de la variación de precios en ámbitos de fuerte demanda. Más alquiler en condiciones contractuales y económicas más inestables ha creado una verdadera burbuja de inseguridad residencial en España, en especial entre los jóvenes, que no disponen de alternativas efectivas. impacto positivo sobre el avance y consolidación de los proyectos vitales de las parejas españolas jóvenes. La modificación reciente de la Ley de Arrendamientos Urbanos podría ir en este sentido, al alargar el plazo de mínimo de los contratos de 3 a 5 años, así como la modificación de la Ley de Enjuiciamiento Civil para ampliar las salvaguardas ante desahucios cuando haya indicios de vulnerabilidad. Establecer medidas que limiten los importes de los alquileres en determinadas áreas urbanas sería lo siguiente. Esperemos que se persevere y se profundice en esta línea.

\section{Referencias bibliográficas}

Bosch, J. (2018) "La burbuja residencial en Europa (2000-2015). Dimensión y tipos". Documents d'Anàlisi Geogràfica, [S.l.]: 1-31.

Eurofound (2017) European Quality of Life Survey 2016: Quality of life, quality of public services, and quality of society, Publications Office of the European Union, Luxembourg.

Leal, J. (2015) "De la burbuja inmobiliaria a los movimientos sociales de los desahuciados". Subirats, J.; García-Bernardos, A., Innovación social y políticas urbanas en España: 120-127.

Lozano, M.; Rentería, E. (2018) “El imparable aumento de los años en precariedad laboral de los adultos jóvenes en España, 1987-2017”. Perspectives demogràfiques, 12: 1-4.

Méndez, R.; Plaza, J. (2016) “Crisis inmobiliaria y desahucios hipotecarios en España: una perspectiva geográfica”. Boletín de la Asociación de Geógrafos Españoles, 71: 99-127.
Módenes, J.A.; López-Colás, J. (2014) "Cambio demográfico reciente y vivienda en España: ¿hacia un nuevo sistema residencial?" Revista Española de Investigaciones Sociológicas (REIS), 148 (1): 103-133

Pareja-Eastaway, M.; Sánchez-Martínez, T. (2017) "Social housing in Spain: what role does the private rented market play?". Journal of Housing and the Built Environment, 32 (2): 377-395

Cita

Juan Antonio Módenes (2019) "El insostenible aumento de la inseguridad residencial en España”. Perspectives Demogràfiques, 13: 1-4.

Editores

Andreu Domingo y Albert Esteve

Correspondencia dirigida a

Juan Antonio Módenes

jamodenes@ced.uab.cat

\section{Créditos}

Gráficos: Anna Turu

Maquetación: Xavier Ruiz Vilchez

Enlace url

http://ced.uab.es/es/difusion/ butlleti-perspectives-demografiques

\section{Agradecimientos}

Algunas de las ideas expresadas en esta contribución aparecen en trabajos previos en coautoría con Julián López-Colás y Alda Azevedo. Deseo expresar mi agradecimiento sincero a los editores Andreu Domingo y Albert Esteve, cuyos comentarios han mejorado el texto. Este artículo se ha realizado en el marco del proyecto del Programa Estatal de Investigación, Desarrollo e Innovación Orientada a los Retos de la Sociedad, CSO2O1679142-R. Se agradece al Centre d'Estudis Demogràfics, institución "CERCA Programme / Generalitat de Catalunya" el apoyo de todos sus miembros para esta publicación.

\section{Contacto}

Centre d'Estudis Demogràfics. Calle de Ca n'Altayó, Edificio E2 Universitat Autònoma de Barcelona o8193 Bellaterra / Barcelona España

Teléfono: +3493581306o E-mail:demog@ced.uab.es Web: http://ced.uab.es/es/

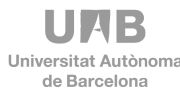

